\title{
Metodologías cooperativas versus competitivas: efectos sobre la motivación en alumnado de EF Cooperative versus competitive methodologies: effects on motivation in PE students
}

\author{
Salvador García Martínez, Pablo Sánchez Blanco, Alberto Ferriz Valero
}

Universidad de Alicante (España)

Resumen. Introducción: El propósito de la presente investigación es la de identificar como los juegos con competición y los juegos cooperativos afectan a la motivación del alumnado en las clases de educación física, en la etapa de la educación secundaria obligatoria. Método: Se ha recopilado la información científica necesaria en bases de datos como Pubmed, Google Scholar, Dialnet, Scopus y Web of Science. Para obtener los datos se ha llevado a cabo el cuestionario de motivación en las clases de educación física (CMEF) en un instituto de educación secundaria obligatoria de la ciudad de Alicante. Resultados: Se observaron mejoras en la motivación intrínseca en todos los grupos de trabajo. También se observaron diferencias significativas en el aumento de la motivación intrínseca del alumnado que realizó los juegos con competición. Conclusiones: Los juegos son una buena herramienta para mejorar la motivación del alumnado y los juegos con competición mejoran significativamente la motivación intrínseca en los estudiantes.

Palabras clave: Juegos cooperativos, juegos competitivos, motivación, educación secundaria.

Summary. Introduction: The purpose of this research is to identify how competitive and cooperative games affect students' motivation in Physical Education classes, at the stage of compulsory secondary education. Method: The necessary scientific information has been collected in databases such as Pubmed, Google Scholar, Dialnet, Scopus, and Web of Science. In order to obtain the data, the motivation questionnaire in Physical Education classes (CMEF) was applied in a compulsory secondary school in the city of Alicante. Results: Intrinsic motivation improvements were observed in all work groups. Significant differences were also observed in the increase of students' intrinsic motivation who performed games with competition. Conclusions: Games are a good tool to improve student's motivation, and competitive games significantly enhance intrinsic motivation in students.

Keywords: Cooperative games, competitive games, motivation, secondary education.

\section{Introducción}

El juego ha tenido un papel muy importante en la historia académica a lo largo del tiempo, tanto como actividad realizada en la práctica educativa como en su desarrollo conceptual. Los profesionales en el área de la educación se han formado de tal manera que el juego ha pasado a ser un aspecto fundamental y una base muy importante de su aprendizaje e instrucción (Pachón, 2016).

Según Extremera y Montero (2016) el juego es un componente educativo muy importante en el proceso de enseñanza-aprendizaje de los niños y niñas sin importar la etapa educativa en la que se encuentren ni en la que se desarrolle. Muchos autores han estudiado el juego en el ámbito educativo y han participado en la ampliación de la definición de juego, en clasificarlos, en exponer sus características y aplicaciones a lo largo de la historia hasta la actualidad. Además, afirman que la utilización del juego como un recurso en la asignatura de educación física tiene que alcanzar una serie de objetivos que permitan y faciliten la óptima ejecución del juego por parte de los alumnos y alumnas. En España, la legislación educativa ha hecho referencia a la importancia del juego en todas las etapas educativas del sistema educativo español.

El juego ayuda al desarrollo de la capacidad creativa y mejora la comprensión de los conceptos y contenidos didácticos de los estudiantes. También facilita el desarrollo de la personalidad de los niños y niñas, diferentes aspectos del carácter, las capacidades físicas y las habilidades sociales del alumnado. El juego también es capaz de generar mu-

Fecha recepción: 09-03-20. Fecha de aceptación: 11-05-20

Salvador García Martínez

salvador.garcia@ua.es chas experiencias que pueden llegar a favorecer su autonomía y adaptación a cualquier situación. Otro de los aspectos importantes del juego que hay que tener en cuenta, es que permite al alumnado a participar de forma activa en las actividades que se proponen por parte del docente con esto se logra un aprendizaje más efectivo y ameno. Aunque el juego es un rasgo distintivo de la infancia, las ganas de jugar perduran a lo largo de la vida de los individuos según Extremera y Montero (2016).

En cuanto al estudio del juego, se empieza a estudiar más a fondo con autores como Claparède, Groos, Elkonin o Chateau y a finales del siglo XX con autores como Piaget, Huizinga Parlebás según Manzano y Carrera (2005).

El presente trabajo pretende observar la motivación del alumnado en las clases de educación física en base al tipo de juego que se realiza. Actualmente, se aconseja por parte de muchos organismos y por muchos profesionales la introducción de juegos de cooperación en las aulas. Autores como Miralles et al. (2017) afirman que el juego cooperativo favorece la participación del alumnado y aumenta la motivación.

\section{Marco teórico}

\section{Definición de juego}

Entre las definiciones más importantes de juego que aparecen en el libro «Componentes básicos del currículum de Educación Física: Casos prácticos para el cuerpo de maestros y profesores» (Extremera y Gallegos, 2015, p. 138), aparece la propuesta por Gross (1902): «El juego es pre-ejercicio de funciones que van a ser necesarias para la vida adulta» 0 Huizinga (1998): «Una acción o actividad voluntaria, realizada dentro de ciertos límites, fijados en el tiempo y en el espacio, que sigue una regla libremente aceptada, pero completa- 
mente imperiosa, provista de un fin en sí misma, acompañada de un sentimiento de tensión y alegría y de una conciencia de ser algo diferente de lo que se es en la vida corriente». Cagigal (1996) lo define como «una acción libre, desinteresada y espontánea que se realiza en una limitación de tiempo y espacio que, además, se encuentra determinada por una serie de normas improvisadas o establecidas». Más adelante, otros autores como Pérez (2016) definen el juego como «un producto puro y espiritual de los niños que transmite y produce alegría, paz, libertad y armonía. Afirman que jugar se define como una acción con la cual podemos producir distracción, placer y entretenimiento a través de la recreación. También concluye que jugar se encuentra en una serie de expresiones culturales como la música, el teatro, la danza, competencias deportivas, juegos de azar y juegos infantiles».

\section{Clasificación de los juegos}

Según la literatura científica, existen diversas formas de clasificar los juegos. Alonso et al. (2013) realizaron la siguiente clasificación, estableciendo cuatro grandes grupos de juegos:

- Juegos psicomotores o individuales. En este tipo de juegos los protagonistas no tienen ningún contrario/adversario que pueda perjudicarle. Tampoco tiene un compañero en el que pueda apoyarse ni recibir ayuda en la realización de cualquier acción. El salto de altura o lanzar una pelota a un objetivo para derribarlo sería un ejemplo de este tipo de juegos.

- Juegos de cooperación. En este tipo de juegos los jugadores deben colaborar entre todos (interacción) para lograr un objetivo final y común. Pasarse una pelota entre los integrantes del equipo sin que se caiga o crear monumentos humanos serían unos ejemplos de este tipo de juegos.

- Juegos de oposición. En este tipo de juegos el jugador tiene un adversario al cual tiene que superar para lograr el objetivo final. Los juegos en los que hay que realizar persecuciones y deportes como el boxeo serían un ejemplo de este tipo de juegos.

- Juegos de cooperación y oposición. En este tipo de juegos varios jugadores tienen que integrar un equipo el cual debe superar a los otros equipos contrarios. Son denominados como «deportes de equipo». Deportes como el fútbol o juegos tradicionales como el cementerio americano serían unos ejemplos de este tipo de juegos.

\section{EI juego en el contexto educativo}

El juego en la asignatura de educación física ha tenido un papel muy importante y una presencia indiscutible a lo largo de toda su historia académica. Su presencia ha sido tanto a nivel conceptual como a nivel práctico siendo una actividad que se lleva a cabo en las sesiones. Para la formación de los docentes en la educación física, el juego ha sido un tema de estudio fundamental (Pachón, 2016).

En los centros e instituciones educativas el poder lúdico es el que más influye en el desarrollo psicológico y social de los estudiantes (Guerrón, 2014). Este autor también asegura que el estar en un aula causa que la explicación de la teoría derive en un estado de pesadez, aburrimiento y sueño. De ahí la importancia que tiene el juego para romper la rutina y refrescar los conocimientos y contenidos que se están tratando. Es importante ir adaptando el juego tanto a las necesidades y edad de los alumnos como a los contenidos y a la recreación del aula.

\section{Juegos cooperativos}

Los juegos cooperativos en la educación no son nada nuevo en la docencia ya que se llevan utilizando durante muchos años. Son muchos los autores que han estudiado y definido estos juegos:

- Teodorico (2004) afirmó que los juegos cooperativos son un conjunto de actividades que hacen posible todos los envueltos de compartir, evaluar y reflejar nuestra relación con nosotros mismos y con los demás. La idea principal de los juegos cooperativos es la de alcanzar un cambio de sensaciones y sentimientos y así entrar en contacto con nuestras propias emociones para potenciar las habilidades humanas como: la libertad, el respeto por los demás, el amor, la creatividad, la paciencia, la humildad, etc. También tienen como propósito el de disminuir las actitudes disruptivas y agresivas en los juegos mediante la incitación de actitudes de cooperación, solidaridad, sensibilización y comunicación.

- Años más tarde, Solana (2007) aseguró que los juegos cooperativos en educación física favorecen el aumento de motivación de todo el alumnado. Además, este tipo de juegos fomentan la cohesión grupal, ayudan al desarrollo cognitivo de los estudiantes y facilita la organización de la clase.

- Otros autores como Curto et al. (2009) y Velázquez (2015) reforzaron la idea de que los juegos cooperativos favorecen la disminución de la ansiedad, aumenta la autoestima y la confianza de los alumnos. También lograr aumentar la motivación y el rendimiento, ayudan a lograr una actitud positiva resultando una mayor implicación en las clases y compromiso en la asignatura de educación física. Otros beneficios de los juegos cooperativos es que aumenta la interacción del alumnado mejorando la comunicación y la inclusión intercultural.

- Para Velázquez (2015), son actividades colectivas donde no hay ni competición ni oposición entre las acciones de los jugadores. Todos buscan lograr un objetivo común, sin importar que tengan los mismos o diferentes papeles. También afirma que el juego cooperativo en educación física es una muy buena herramienta para transmitir y formar buenos valores en los estudiantes.

- Según Miralles et al. (2017) los juegos cooperativos ofrecen una gran variedad de circunstancias y situaciones en las cuales aparecen conductas relacionadas con la comunicación, el sacrificio, la cooperación, el pacto, la toma de iniciativa, crear respuestas creativas y originales y respetar las decisiones de sus compañeros y la voluntad del grupo.

\section{Juego cooperativo no es aprendizaje cooperativo}

Como puede crear confusión, es importante insistir en que el juego cooperativo no es aprendizaje cooperativo. Velázquez (2018) nos expone algunas diferencias entre el juego y el aprendizaje cooperativo:

El juego cooperativo es una actividad con un carácter específico y puntual. En cambio, el aprendizaje cooperativo es una metodología, una práctica pedagógica que se prolon- 
ga en el tiempo.

La finalidad que persiguen es diferente: el juego busca la diversión de los jugadores mientras el docente lo utiliza para alcanzar objetivos didácticos; el aprendizaje cooperativo busca que los estudiantes aprendan, sin importar lo divertido y entretenido que sea el proceso.

El método de evaluación es distinto: en el caso del aprendizaje cooperativo, la autoevaluación grupal es un elemento fundamental, pero en el caso del juego cooperativo solo es aconsejable.

El nivel de responsabilidad que tiene el alumnado no es el mismo: en el aprendizaje cooperativo la responsabilidad individual es ineludible ya que no se puede alcanzar el objetivo final si alguno de los integrantes del grupo se escuda en el trabajo de los demás; en el juego cooperativo solo se necesita la responsabilidad grupal, donde un jugador puede tomar una actitud pasiva durante la realización de la actividad sin que esto afecte de forma negativa a la consecución del objetivo común.

\section{La competición}

El diccionario de la Real Academia de la Lengua Española (2001) define competición como: «competencia o rivalidad de quienes se disputan una misma cosa o la pretenden». En el ámbito del deporte lo define como: «la acción o efecto de competir».

Hay muchos estudios científicos como los de Cecchini et al. (2008) y Sánchez-Oliva et al. (2012) donde se asegura que la capacidad competitiva en el deporte es un elemento que genera motivación, compromiso, satisfacción y adherencia hacia el deporte. También afirman que la competición es una fuente de desarrollo social y personal.

\section{La motivación}

Según Roberts (1995) y Dosil (2004) la motivación es un concepto pluridimensional, indefinido y que es capaz de explicar el comportamiento de los seres humanos. Estos autores han destacado tres elementos constitutivos de la motivación: la dirección, la intensidad y la persistencia de la conducta. Años más tarde, Weinberg y Gould (2018) consideran que la motivación es una herramienta psicológica que tiene el poder sobre la dirección, persistencia e intensidad de la conducta de todas las personas. La dirección hace referencia a los objetivos que se propone una persona, la intensidad se refiere a la cantidad de energía y esfuerzo que se emplea para lograr dichos objetivos y la persistencia al tiempo que lleva a cabo el sujeto para la consecución de los mismo.

Zamarripa et al. (2016) afirmaron que una persona puede motivarse de tres formas: de modo intrínseco, extrínseco y puede no estar motivada. Estos tres tipos de motivación cambian en su grado de autonomía, pudiendo situarse en un continuo de la autonomía donde la conducta puede ir variando. La conducta puede ir cambiando desde unos niveles muy altos de autonomía (motivación intrínseca), pasando por unos niveles intermedios (motivación extrínseca), hasta unos niveles bajo (no hay motivación).

En esta investigación se ha cuantificado y estudiado la motivación intrínseca del alumnado de educación física. Según Menéndez y Fernández-Río (2017), se habla de motiva- ción intrínseca cuando una persona está motivada al realizar una acción o actividad por la única razón de disfrutar de la mismas sin ningún tipo de interés distinto al placer personal y realizarla de forma exitosa. Las actividades que promueven este tipo de motivación suelen ser actividades entretenidas y divertidas capaces de desarrollar sensaciones de autodeterminación y competencia. Las actividades divertidas motivan al alumnado y suscita gran interés en ir mejorando poco a poco.

Existen diversas investigaciones que señalan que la motivación en educación física provoca: un aumento del esfuerzo en las clases de educación física y la intención de ser físicamente activo fuera del instituto (Méndez-Giménez et al., 2012); aumenta el tiempo de práctica deportiva fuera del ámbito escolar (Moreno et al. 2009).

Por tanto, el presente estudio tiene como objetivo general, identificar como afectan los juegos, en función de sus características y tipología, en la motivación intrínseca del alumnado en las clases de educación física, en la etapa de la educación secundaria obligatoria. De manera específica, se plantean los siguientes objetivos de la investigación: Seleccionar la información científica necesaria para fundamentar los contenidos del marco teórico, analizar y cuantificar la motivación intrínseca del alumnado que practica juegos cooperativos en las clases de educación física y analizar y cuantificar la motivación intrínseca del alumnado que practica juegos con competición en las clases de educación física.

El presente trabajo presenta tres hipótesis: 1. El alumnado que lleva a cabo juegos con competición aumenta significativamente la motivación intrínseca por encima de los alumnos y alumnas que participan en juegos de cooperación; 2. el alumnado que lleva a cabo juegos cooperativos aumenta significativamente la motivación intrínseca por encima de los alumnos y alumnas que participan en juegos con competición. 3. La motivación intrínseca del alumnado que realiza las sesiones de juegos cooperativos y juegos con competición no va a presentar diferencias significativas.

\section{Método}

La presente investigación es un estudio empírico que usa metodología de carácter cuantitativa con un diseño cuasi experimental por conglomerados no probabilístico intra e inter-grupos con medidas de prueba antes y después de la intervención. Se ha realizado la identificación y análisis de una problemática para observar la motivación del alumnado en las clases de educación física con juegos competitivos y juegos cooperativos. La recogida de datos se ha llevado a cabo en un instituto de educación secundaria obligatoria de Alicante.

\section{Participantes}

En este estudio han participaron un total de 45 alumnos ( 27 chicas y 18 chicos) de Educación Secundaria Obligatoria de un instituto de Alicante. Manteniendo la naturaleza de los grupos, se les aplicaron un muestreo por conglomerados no probabilístico, dando lugar a dos grupos formados por tres clases de segundo curso de educación secundaria obligatoria. La edad de los participantes era de media 13.8 años ( \pm .68 años). 
La muestra fue dividida en tres grupos de trabajo. El primer grupo (grupo experimental 1) estaba compuesto por alumnado de $2^{\circ} \mathrm{A}$ de ESO que contaba con 14 estudiantes (ocho chicas y seis chicos) y realizaron una serie de juegos y actividades con competición. El segundo grupo (grupo experimental dos estaba formado por alumnado de $2^{\circ} \mathrm{B}$ de ESO que contaba con 14 estudiantes (nueve chicas y cinco chicos) y realizaron los juegos cooperativos. El tercer grupo (grupo control) estaba compuesto por alumnado de $2^{\circ} \mathrm{C}$ de ESO que contaba con 17 estudiantes (10 chicas y siete chicos) y realizaron la unidad didáctica de voleibol. La intervención fue llevada a la práctica por una persona licenciada en 'Ciencias de la actividad física y el deporte' (profesorado de Educación Física).

\section{Instrumentos}

Para llevar a cabo esta investigación se empleó, como herramienta para obtener la información necesaria, un cuestionario validado. Este cuestionario nos ha servido para extraer los datos para medir la motivación del alumnado en cada grupo de trabajo. El cuestionario que se ha utilizado es un cuestionario de motivación en las clases de educación física (CMEF) de Sánchez-Oliva et al. (2012). Dicho cuestionario contiene un total de 20 ítems, los cuales deben ser respondido mediante una escala Likert en referencia al grado de conformidad del encuestado con las siguientes posibles respuestas:

- Totalmente en desacuerdo.

- Algo en desacuerdo.

- Neutro.

- Algo de acuerdo.

- Totalmente de acuerdo.

Este cuestionario mide los diferentes tipos de motivación, cada ítem responde a un tipo específico. Los ítems 1, 6, 11 y 16 corresponden a la motivación intrínseca; 2, 7, 12 y 17 para la regulación identificada; $3,8,13$ y 18 para la regulación introyectada; 4, 9, 17 y 19 para la regulación externa y, por último, los ítems 5, 10, 15 y 20 para la desmotivación. En el caso de esta investigación, se ha analizado los ítems respectivos a la motivación intrínseca.

Este estudio es de carácter descriptivo y transversal. Las variables del estudio son:

- Variables dependientes: Motivación Intrínseca.

- Variables independientes: Edad, Género y Curso.

\section{Procedimiento}

Una vez decidida la línea de investigación y obtenido los permisos por parte del centro educativo y las familias del alumnado participante, se realizó la búsqueda de un cuestionario validado y relacionado con la investigación que se iba a llevar a cabo. Se decidió no realizar ninguna modificación para evitar cualquier tipo de problema en la obtención e interpretación de los datos obtenidos. En consecuencia, se explicó en profundidad y detalladamente el cuestionario y se realizaron las aclaraciones necesarias.

La intervención tuvo una duración de dos semanas para todos los grupos donde se llevaron a cabo las actividades propuestas. La realización de los cuestionarios por parte del alumnado de los tres grupos se efectuó al principio (pre-test) $\mathrm{y}$ al final (post-test) de la investigación (Tabla 1).

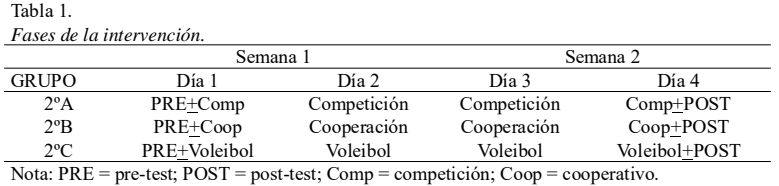

Todos los grupos de trabajo realizaron sus respectivas actividades, en función del grupo de trabajo, a lo largo de las dos semanas. El primer día (día primero), se dividió la sesión en dos partes para todos los grupos $\left(2^{\circ} \mathrm{A}, 2^{\circ} \mathrm{B}\right.$ y $2^{\circ} \mathrm{C}$ de ESO $)$ : en la primera parte seguían trabajando los juegos propuestos por el investigador y la unidad didáctica por parte del grupo control; y la segunda parte constaba de 20 minutos en la cual se explicaba detenidamente el cuestionario por parte del docente durante 10 minutos y se dejaron otros 10 minutos para que los estudiantes respondieran los 20 ítems del cuestionario (CMEF) y lo completaran. El último (día cuarto), el alumnado volvió a realizar el cuestionario en los últimos 15 minutos de clase.

\section{Resultados}

Para el análisis de los resultados obtenidos, se hizo uso del programa estadístico Statistics Product and Service Solutions (IBM ${ }^{\circledR}$ SPSS $^{\circledR}$ Statistics Versión 26.0.0.0) y Microsoft Excel ${ }^{\circledR}$ en su versión 2016. Mediante estadísticos descriptivos (media y desviación típica) se describen los resultados relativos a las variables del estudio; suma pre y post (suma de los ítems 1, 6, 11 y 16). El análisis de significación estadística de cada curso y el género se realizó mediante la prueba de rango con signo de Wilcoxon para muestras relacionadas. El nivel de significación estadística se ha fijado en $p<.05$.

Se procede en primer término analizar la normalidad de los datos y, dado que la muestra es menor que $50(\mathrm{~N}=45)$, se utilizado los datos de Shapiro-Wilk, obteniendo que para los tres cursos no se cumple la normalidad y se debe realizar un análisis a través de una prueba no paramétrica.

\section{Diferencias de partida de los grupos}

A través de la prueba no paramétrica de U de MannWhitney, se analizaron si existían diferencias en los resultados de los cuestionarios iniciales para detectar posibles diferencias de partida, no obteniendo significatividad estadística $(p<.05)$ en los mismos tal y como aparecen en la tabla 2 , lo que lleva a concluir a partir de dicho resultado, que no hay diferencias de motivación de partida, es decir, se parte del mismo nivel de motivación.

Tabla 2.
\begin{tabular}{lcc} 
Resultados de la prueba no paramétrica de U de Mann-Whitney del cuestionario inicial CMEF. \\
\hline U de Mann-Whitney & SUMA PRE & SUMA POST \\
gl & 2,907 & 8,176 \\
Sig. asintótica & 2 & 2 \\
\hline
\end{tabular}

\section{Diferencias en la media Pre-Post}

Se observa en la tabla 3, como en todos los grupos aumenta la motivación intrínseca una vez realizada la intervención. Los cursos donde más aumenta la motivación intrínseca son en aquellos donde se han realizado los juegos $\left(2^{\circ} \mathrm{A}\right.$ y $2^{\circ} \mathrm{B}$ de ESO). En el grupo control, aumenta, pero en menor medida $\left(2^{\circ} \mathrm{C}\right.$ de ESO $)$. 
Tabla 3.

\begin{tabular}{|c|c|c|c|}
\hline & $2^{\circ} \mathrm{A}$ ESO & $2^{\circ} \mathrm{B}$ ESO & $2^{\circ} \mathrm{C}$ ESO \\
\hline Media Pre+DE & $16.14 \pm 1.87$ & $15.71 \pm 1.89$ & $14.82 \pm 2.09$ \\
\hline Media Post+DE & $17.57 \pm 2.10$ & $16.64 \pm 2.37$ & $15.06 \pm 1.95$ \\
\hline $\mathrm{N}$ & 14 & 14 & 17 \\
\hline
\end{tabular}

\section{Diferencias por cursos}

A continuación, en la tabla 4 se muestra las diferencias entre los cursos. Una vez analizado los datos, los resultados señalan que existen mejoras estadísticamente significativas $(p<.05)$, solo en el curso de $2^{\circ} \mathrm{A}$ ESO (juegos de competición).

\begin{tabular}{|c|c|c|}
\hline Clase & & suma post - suma pre \\
\hline \multirow{2}{*}{$2^{\circ} \mathrm{A}$ ESO } & Z & $-2.291 \mathrm{c}$ \\
\hline & Sig. asintótica(bilateral) & .022 \\
\hline \multirow{2}{*}{$2^{\circ} \mathrm{B}$ ESO } & $\mathrm{Z}$ & $-1.437 \mathrm{c}$ \\
\hline & Sig. asintótica(bilateral) & .151 \\
\hline \multirow{2}{*}{$2^{\circ} \mathrm{C}$ ESO } & Z & $-.246 c$ \\
\hline & Sig. asintótica(bilateral) & .805 \\
\hline
\end{tabular}

\section{Diferencias por género}

Para observar diferencias por género, se ha diferenciado la muestra $(\mathrm{N}=45)$ entre alumnado masculino y alumnado femenino, obteniendo mejora estadísticamente significativa $(p<.05)$ en el género masculino de la clase de $2^{\circ} \mathrm{A}$ y se observa una tendencia a la significatividad en el género femenino del mismo grupo. Por otro lado, los resultados señalan que no existen mejoras estadísticamente significativas, en ninguno de los géneros de los demás grupos, en la motivación intrínseca. Esto se debe a que, en ningún caso, la significación de los resultados ha sido $p<.05$. Tabla 5 .

Tabla 5 .

Prueba de Wilcoxon para muestras relacionadas desglosado por género.

\begin{tabular}{lccc}
\multicolumn{1}{c}{ Clase } & Género & suma post - suma_pre \\
\hline $2^{\circ} \mathrm{A}$ ESO & $\mathrm{M}$ & .022 \\
& $\mathrm{~F}$ & .062 \\
$2^{\circ} \mathrm{B}$ ESO & $\mathrm{M}$ & \multirow{2}{*}{ Sig. asintótica(bilateral) } & .109 \\
& $\mathrm{~F}$ & .595 \\
$2^{\circ} \mathrm{C}$ ESO & $\mathrm{M}$ & .785 \\
& $\mathrm{~F}$ & .621 \\
\hline Nota: M: masculino; F: Femenino. &
\end{tabular}

\section{Discusión}

El objetivo de la presente investigación es la de identificar como los juegos con competición y los juegos cooperativos afectan a la motivación del alumnado en las clases de educación física tradicional, en la etapa de la educación secundaria obligatoria. En este sentido, los datos y resultados obtenidos en la investigación muestran resultados positivos de juego como adecuada herramienta para mejorar la motivación del alumnado, en este caso, para aumentar la motivación intrínseca. Además, cabe destacar que los tres grupos de investigación partían de niveles de motivación similares sin diferencias entre los mismos.

Así pues, el resultado más relevante de la presente investigación, aparece en los grupos de trabajo donde se ha impartido sesiones con juegos como herramienta para mejorar la motivación $\left(2^{\circ} \mathrm{A}\right.$ y $2^{\circ} \mathrm{B}$ de ESO $)$ ya que ha aumentado de manera específica la motivación intrínseca e incluso en el grupo A esta mejora ha sido muy positiva desde el ámbito educativo por la significatividad de los resultados, en cambio, en el grupo control $\left(2^{\circ} \mathrm{C}\right.$ de ESO $)$ ha aumentado, pero en menor medida. Estos resultados coinciden con los obtenidos por Granero-Gallegos y Baena-Extremera (2014) que se- ñalan que la motivación está influenciada por las características de las actividades que se plantean. Estos autores observaron que mediante tareas diferentes y divertidas que lograran atraer a los alumnos y alumnas, se obtienen mejoras en la motivación intrínseca de los sujetos analizados. En la misma línea, García (2017) en otro estudio realizado en la clase de educación física con alumnado de noveno grado, concluyó que la implementación de un programa de actividades físicas acuáticas compuesto por juegos libres, dirigidos y cooperativos afectó positivamente a la motivación intrínseca de los sujetos analizados, a lo que corrobora los resultados obtenidos en la presente investigación.

Con estos resultados podemos corroborar la primera hipótesis de la investigación, es decir, el alumnado que lleva a cabo juegos competitivos aumenta significativamente la motivación intrínseca por encima del alumnado que participa en juegos de cooperativos.

Para Amado et al. (2014) existen diferencias significativas en cuanto a la necesidad de competición en el género masculino. Los hombres se sienten más competentes, para realizar deporte, que las mujeres. Estos resultados también han sido respaldados anteriormente por otros autores (Moreno et al., 2007) pero con alumnado de educación primaria. Hay que tener en cuenta apenas se han encontrado estudios científicos en esta línea de investigación. Sería interesante investigar sobre la motivación del alumnado en los juegos competitivos y en los juegos cooperativos en las clases de educación física.

Otro aspecto para destacar en la presente investigación, hace referencia la diferencia por género en la motivación intrínseca, una vez realizada la búsqueda de información científica, se ha observado que no existe una unanimidad entre los investigadores con relación a la motivación intrínseca y el género. Según Amado et al (2014), los resultados de su análisis demostraron que los chicos presentan puntuaciones más altas que las chicas en la motivación intrínseca. En cambio, otros autores como Cuevas et al. (2016) encontraron diferencias significativas a favor de las alumnas en la motivación intrínseca en las clases de educación física. En el presente estudio los resultados obtenidos coinciden con el primer autor se han encontrado mejoras estadísticamente significativas en los chicos del grupo experimental $2^{\circ} \mathrm{A}$ y una tendencia en las chicas.

Cabe destacar, que la presente investigación presenta la limitación de un tamaño de la muestra reducido y una muestra intencional, pero no por ello lleva a desechar los resultados obtenidos para la necesidad de formar al profesorado en este tipo de temática. Con lo que se propone continuar con este tipo de estudios desde el ámbito de la educación física para mejorar el interés hacia la actividad deportiva o física y de manera específica ahondar en los estudios de NavarroPatón et al. (2018) en la incidencia de los programas basado en juegos cooperativos en la mejora del autoconcepto físico de los estudiantes en la asignatura de educación física.

\section{Conclusiones}

Para finalizar con la investigación se extraen las siguientes conclusiones:

- Los juegos son una buena herramienta para lograr 
que los alumnos y alumnas aumenten su motivación intrínseca. Mediante la utilización de actividades lúdicas que sean interesantes y divertidas para los estudiantes, podemos aumentar la motivación de la clase en la asignatura de educación física.

- Los juegos con competición mejoran de forma significativa la motivación intrínseca del alumnado. El factor competición es fundamental para lograr una significación positiva en el aumento de la motivación del alumnado.

- Respecto al género, no se mejora significativamente la motivación intrínseca ni de hombres ni mujeres mediante los juegos.

\section{Referencias}

Alonso, J.I., Gea, G., \& Yuste, J.L. (2013). Formación emocional y juego en futuros docentes de Educación física. Revista Electrónica Interuniversitaria de Formación del Profesorado, 16(1), 97108. https://doi.org/10.6018/reifop.16.1.179461

Amado, D., Sánchez-Miguel, P.A., Leo, F.M.; Sánchez-Oliva, D. \& García-Calvo, T. (2014) Diferencias de género en la motivación y percepción de utilidad del deporte escolar. Revista Internacional de Medicina y Ciencias de la Actividad Física y el Deporte, 14(56), 651-664. Recuperado de https://repositorio.uam.es/ b i t s t r e a m/hand le/10486/662999/ RIMCAFD_56_5.pdf? sequence $=1 \&$ isAllowed $=\mathrm{y}$

Cagigal, J.M. (1996). Obras Selectas. Cádiz, España: Comité Olímpico Español.

Cecchini, J., Fernández, J., González, C., \& Arruza, J. (2008). Repercusiones del Programa Delfos de educación en valores a través del deporte en jóvenes escolares. Revista de Educación, 346, 167-186. Recuperado de https://www.redined.mepsyd.es/xmlui/ b i t s t e a m/hand le / $11162 / 72039 /$ 00820083000173.pdf? sequence $=1 \&$ is Allowed $=\mathrm{y}$

Cuevas, E., Pérez, G., \& Torres, B. (2016). Análisis de la motivación en las clases de Educación Física en Primaria. E-motion: Revista de Educación, Motricidad e Investigación, (7), 3-12. Recuperado de http://rabida.uhu.es/dspace/bitstream/handle/10272/13972/ Análisis.pdf? sequence $=2$

Curto, C.; Gelabert, I.; González, C. \& Morales, J. (2009). Experiencias con éxito de aprendizaje cooperativo en Educación Física. Barcelona, España.

Dosil, J. (2004). Psicología de la actividad física y del deporte. Madrid, España: Mac Graw Hill.

Extremera, A. B., \& Gallegos, A. G. (2015). Componentes básicos del currículum de Educación Física: Casos prácticos para el cuerpo de maestros y profesores. Wanceulen Editorial SL.

Extremera, A., \& Montero, P. (2016). El juego motor como actividad física organizada en la enseñanza y la recreación. EmásF: revista digital de educación física, (38), 73-86. Recuperado de https://www.researchgate.net/profile/Antonio_Extremera/ publication328789470_EL_JUEGO_MOTOR_COMO_ACTIVIDAD_FISICA ORGANIZADA_EN_LA_ENSENANZA_Y_LA_RECREACION/ links/5be307dea6fdcc3a-̄dc50bdb/EL-JUEGO-MOTOR-COMOACTIVIDAD-FISICA-ORGANIZADA-EN-LA-ENSENANZAY-LA-RECREACION.pdf

García C. (2017). Efecto de un programa de ejercicio físico acuático sobre la motivación hacia la clase de educación física en estudiantes de noveno grado de un colegio de Cimitarra, Santander. Revista salud historia sanidad, 12(1), 69-79. https://doi.org/10.1909/ shs.v12i1.170

Granero-Gallegos, A. \& Baena-Extremera, A. (2014). Predicción de la motivación autodeterminada según las orientaciones de meta y el clima motivacional en Educación Física. Retos. Nuevas tendencias en Educación Física, Deporte y Recreación, 25, 23-27. Recuperado de https://www.redalyc.org/pdf/3457/ 345732291005.pdf

Gross, K. (1902). The play of animals. New York, EEUU: Appleton. Guerrón, D. (2014). Las actividades lúdicas en el aprendizaje de cultura física en la Unidad Educativa «Juan Pablo II» del cantón Ibarra provincia de Imbabura durante el periodo 20122013. Propuesta alternativa (Tesis doctoral). Universidad Téc- nica del Norte, Ibarra, España.

Huizinga, J. (1998). Homo ludens: el elemento lúdico de la cultura. Madrid, España: Alianza.

Manzano, J.I. \& Carrera, I. (2005). La E.F. en el proceso educativo. Madrid, España: Infornet.

Méndez-Giménez, A., Cecchini, J. A., Fernández-Río, J., \& González, C. (2012). Autodeterminación y metas sociales: un modelo estructural para comprender la intención de práctica, el esfuerzo y el aburrimiento en educación física. Aula Abierta, 40(1), 51-62. Recuperado de http://digibuo.uniovi.es/dspace/bitstream/10651/ 8883/1/AulaAbierta.2012.40.1.51-62.pdf

Menéndez, J. I., \& Fernández-Río, J. (2017). Responsabilidad social, necesidades psicológicas básicas, motivación intrínseca y metas de amistad en educación física. Retos: nuevas tendencias en educación física, deporte y recreación, (32), 134-139. Recuperado de https://www.redalyc.org/pdf/3457/345751100027.pdf

Miralles, R., Filella, G., \& Lavega, P. (2017). Educación física emocional en el ciclo superior de educación primaria. Ayudando a los maestros a tomar decisiones. Retos: nuevas tendencias en educación física, deportes y recreación, (31), 88-93. Recuperado de https://repositori.udl.cat/bitstream/handle/10459.1/59188/ 025046.pdf? sequence $=1$ \& is Allowed $=y$

Moreno, J. A., López de San Román, M., Martínez, C., Alonso, N., \& González-Cutre, D. (2007). Efectos del género, la edad, y la frecuencia de práctica en la motivación y el disfrute del ejercicio físico. Fitness and Performance Journal, 6(3), 140-146.

Moreno, J. A., Zomeño, T., Marín, L. M., Cervelló, E., \& Ruiz, L. M. (2009). Variables motivacionales relacionadas con la práctica deportiva extraescolar en estudiantes adolescentes de educación física. Apunts. Educación Física y Deporte, 95, 38-43.

Navarro-Patón, R., Ferreira, B. R., \& García, M. G. (2018). Incidencia de los juegos cooperativos en el autoconcepto físico de escolares de educación primaria. Retos, 34, 14-18.

Pachón, J. (2016). El juego en el ámbito disciplinar de la educación física y la formación de sus docentes. Lúdica Pedagógica, 24, $79-88$.

Pérez, F. (2016). Aplicación de herramientas de gamificación en física y química de secundaria. Opción: Revista de Ciencias $\mathrm{Hu}$ manas y Sociales, (12), 327-348. Recuperado de https:// www.researchgate.net/profile/Felipe_Quintanal/publication/ 311767939 Aplicacion_de_herramientas_de _gamificacion_en_fisica_y_quimica_de_secundaria/links/ 58599f4608ae64cb3 d4943a1/Aplicacion-de-herramientas-degamificacion-en-fisica-y-quimica-de-secundaria.pdf

Real Academia Española. (2001). Disquisición. En Diccionario de la lengua española (22.a ed.). Recuperado de http:// $b u s \quad c \quad o \quad n$. $r a e^{2}$. e s / d r a e I / SrvltConsulta?TIPO_BUS $=3 \& L E M A=$ disquisici\%F3n

Roberts, G. (1995). Motivación en el deporte y el ejercicio. Bilbao, España: Desclée De Brouwer.

Sánchez-Oliva, D., Sánchez-Miguel, P. A., Leo, F. M.; Amado, D., \& García-Calvo, T. (2013). Desarrollo y validación de un cuestionario para analizar la percepción de comportamientos positivos en las clases de educación física. Cultura y Educación, 25(4), 495-507. https://doi.org/10.1174/113564013808906843

Solana, A. (2007). Aprendizaje cooperativo en las clases de educación física. Sevilla, España: Wanceulen.

Teodorico, M. (2004). Los juegos cooperativos en la educación física: una propuesta lúdica para la paz. Revista Tándem, 14, 21- 31.

Velázquez, C. (2015). Aprendizaje Cooperativo en Educación Física: estado de la cuestión y propuesta de intervención. Retos, 28, 234-239. Recuperado de https://www.redalyc.org/pdf/3457/ 345741428042.pdf

Velázquez, C. (2018). El aprendizaje cooperativo en educación física: Planteamientos teóricos y puesta en práctica. Acción motriz, (20), 7-16. Recuperado de http://www.accionmotriz.com/documentos/revistas/articulos/20_1.pdf

Weinberg, R., \& Gould, D. (2018). Foundations of Sport and Exercise Psychology, 7E. Champaign, Illinois, United States: Human Kinetics.

Zamarripa, J., Castillo, I., Tomás, I., Tristán, J., \& Álvarez, O. (2016). El papel del profesor en la motivación y la salud mental de los estudiantes de educación física. Salud mental, 39(4), 221-227. Recuperado de http://www.scielo.org.mx/pdf/sm/v39n4/01853325-sm-39-04-00221.pdf 